\title{
康布瑞汀磷酸酯二钠盐合成工艺的优化
}

\author{
肖繁花吴范宏* \\ (上海应用技术学院化学与环境工程学院 上海 201418)
}

\begin{abstract}
摘要 对康布瑞汀磷酸酯二钠盐的合成工艺进行优化. 确定了以 $3,4,5$-三甲氧基苯甲醛为原料, 经还原、澳代、成鏻盐 合成中间体 3, 4, 5-三甲氧基芐溴三苯基鏻盐; 鏻盐与 3-三苯甲氧基-4-甲氧基苯甲醛经 Wittig 反应、脱三苯甲基保护基、 与亚磷酸二芐酯成酯、脱芐基成钠盐得到康布瑞汀磷酸酯二钠盐的方法. 所得康布瑞汀磷酸酯二钠盐(1), HPLC 纯度达 99.5\%. 总收率为 $43.0 \%$. 经优化后的工艺原料价廉易得, 操作简便, 成本低廉, 适合于工业化生产.
\end{abstract}

关键词 康布瑞汀磷酸酯二钠; 抗癌药; 合成

\section{Process Optimization for the Synthesis of Disodium Combretastatin A-4 Phosphate}

\author{
Xiao, Fanhua $\quad$ Wu, Fanhong* \\ (School of Chemical and Environmental Engineering, Shanghai Institute of Technology, Shanghai 201418)
}

\begin{abstract}
The method for the synthesis of disodium combretastatin A-4 phosphate was studied. The target product was prepared from 3,4,5-trimethoxyl benzaldehyde as the starting material, through 7-step reactions including reduction, bromation, phosphonium salt formation, Wittig reaction, detritylation reaction, dibenzylphosphorylation reaction, deprotection and disodium salt formation. Disodium combretastaton A-4 phosphate (1) was prepared with HPLC purity of $99.8 \%$ and the total yield of $43.0 \%$. The optimized synthetic process has the advantages of available starting material, simplicity and cheapness, which was more suitable for industrialized production.
\end{abstract}

Keywords disodium combretastatin A-4 phosphate; anti-cancer drug; synthesis

康布瑞汀磷酸酯二钠盐(combretastatin A4 phosphate, 简称 CA4P, 1) 是抗肿瘤药 CA4 的一种前 药 ${ }^{[1 ~ 3]}$. 由美国 OXIGENE 公司开发的新型抗肿瘤前体 药物, 其原药为 CA4 (Combretastatin A4), 系从非洲灌 木 Combretum Caffrum 的树皮中分离得到的一种多羟基 二苯乙烯类天然产物. CA4 是微管蛋白结合剂(目前最 为有限的一类抗癌药物), 能干扰肿瘤血管内皮细胞的 有丝分裂过程，具有很强的抗血管效应、抑制癌细胞增 长、抑制微管蛋白聚集及放化疗增敏作用, CA4 还因其 前体化设计而具有适合临床应用的良好药代动力学性 质并对瘤体呈现靶向性, CA4 对所有癌症模型均显示体 内活性, 被誉为血管靶向剂(Vascular targeting agent, VTA)中的佼佼者, 是全球范围内第一个进入临床研究 的 $\mathrm{VTA}^{[4]}$. 目前 CA4 正在美国和欧洲进行III期临床试 验, 且已获准进入美国食品及药品管理局(FDA)快通道 审批. 由于 CA4 的水溶性很差, 不易被人体吸收, 而且
毒副作用大，使它的应用受到很大的限制. 在对其结构 修饰研究中发现, CA4 的磷酸二钠盐 CA4P 是一种水溶 性前药分子，使它更易于体内给药 ${ }^{[5,6]}$.

据文献和专利报道 CA4P 的合成主要分为 CA4 的 合成和由 CA4 合成 CA4P 两部分. CA4 的合成关键一步 形成二苯乙烯结构的双键, 主要有 Perkin 反应、Wittig 反应、Suzuki 反应等方法 ${ }^{[7 \sim 9]}$. 通过对各种方法比较, 本 文选择了适合于工业化的 Wittig 反应方法. 以 3,4,5-三 甲氧基苯甲醛为原料，经还原、溴代、成鏻盐合成中间 体 3,4,5-三甲氧基芐溴三苯基鏻盐(7); 鏻盐与 3-三苯甲 氧基-4-甲氧基苯甲醛(2)经 Wittig 反应、脱三苯甲基保 护基得到 CA4 (9). 由 CA4 磷酰化合成 CA4P 所用的试 剂有亚磷酸二芐酯、双-(2,2,2-三氯乙基)磷酰氯芐、烷 基胺磷化物等 ${ }^{[10 ~ 12]}$. 本文采用自制的亚磷酸二芐酯作 为磷酰化试剂. 通过对工艺进行改进和优化, 确定了一 条适合于工业化生产的合成 CA4P 的路线. 总收率达到

* E-mail: wfh@sit.edu.cn

Received January 16, 2013; revised February 21, 2013; published online March 7, 2013. 
43.0\%. 合成路线如 Scheme 1 所示.

\section{1 结果与讨论}

最后一步磷酰化反应所用试剂亚磷酸二芐酯(DBP) 国内没有生产和出售, 进口试剂非常昂贵. 在亚磷酸二 芐酯的制备中, 采用了价廉易得的原料; 提纯使用快速 过柱的方法得到了含量 $85 \%$ 的 DBP, 在磷酰化反应中与 进口的 DBP 比较, 得到完全一致的效果. 大幅度减低了 最后一步的原料成本.

在异香草醛的羟基保护反应中, 文献 $[4,8]$ 采用与 价格昂贵的叔丁基二甲基氯硅烷反应生成硅醚的方法, 不仅成本高，而且反应条件苛刻，不利于工业化生产. 我们参考文献[13], 使用三苯甲基氯为保护试剂, 使用 价廉易得的原料和溶剂和常规操作, 得到中等产率和纯 度较高的异香草醛保护体 $\mathbf{3}$.

在中间体 5 的合成中, 采用价廉的硼氢化钾代替价 高的硼氢化钠, 嗍氢化钾与 3,4,5-三甲氧基苯甲醛的摩 尔比由文献的 $0.44: 1$ 下降到 $0.30: 1$, 采用先浓缩、再 加水和二氯甲烷萃取的后处理方法, 还原反应的收率接 近 100\%. 在鏻盐 7 的合成中, 粗品精制文献[13]采用甲 苯、丙酮分别洗涤 2 次的方法, 但文献没有提供鏻盐的 纯度数据. 我们按照文献[13]操作, 发现得到的鏻盐中 夹杂了较多的杂质, 纯度为 $87.5 \%$. 为此我们采用了将 粗品用乙醇重结晶的方法, 使鏻盐的纯度达到 $98 \%$ 以
上.

化合物 8 的制备 Wittig 反应是整个工艺中的关键一 步. 反应中形成的双键有顺反异构体, 研究发现反应物 的物质的量比、反应温度等对反应产物中顺反异构体的 比例有很大的影响. 通过实验确定了反应物物质的量比 为: 鏻盐 7 : 异香草醛保护体 $3:$ 正丁基锂 $=1: 1: 1.2$; 反应温度为 $-20 \sim-15{ }^{\circ} \mathrm{C}$; 得到的化合物 8 的顺反异 构体的比例为 $6.1 \sim 7.2: 1$.

化合物 9 的合成是在强酸性条件下醚键的脱保护水 解反应. 文献[13]报道在甲苯中与浓盐酸反应, 粗品经 甲苯三次淋洗, 收率为 $45 \sim 65 \%$, 也没有化合物 9 的纯 度数据. 按照文献操作, 我们发现, 不仅 9 的收率很低 (44\%), 而且 HPLC 含量只有 $75 \%$ 左右，同时我们在水层 发现还有很多产品, 但纯度很低(HPLC含量 $50 \%$ ). 我们 将反应溶剂由甲苯改为乙醇，不仅简化了后处理步骤， 而且大大提高了产品的纯度和反应的收率.

在 CA4 与亚磷酸二芐酯的磷酰化反应中, 考察了 亚磷酸二苄酯与化合物 9 的投料比对反应的影响. 文献 中亚磷酸二芐酯与化合物 9 的物质的量比为 $1.42: 1$, 过量的亚磷酸二芐酯不仅造成了很大的浪费, 而且没有 反应的亚磷酸二芐酯会夹杂在产品中, 不易去除, 导致 化合物 10 的精制难度增加和反应收率的降低. 实验表 明亚磷酸二芐酯与化合物 9 的最佳物质的量比为 1.1 :

1. 得到粗品中除了少量未反应的原料外, 通常含有 $5 \%$

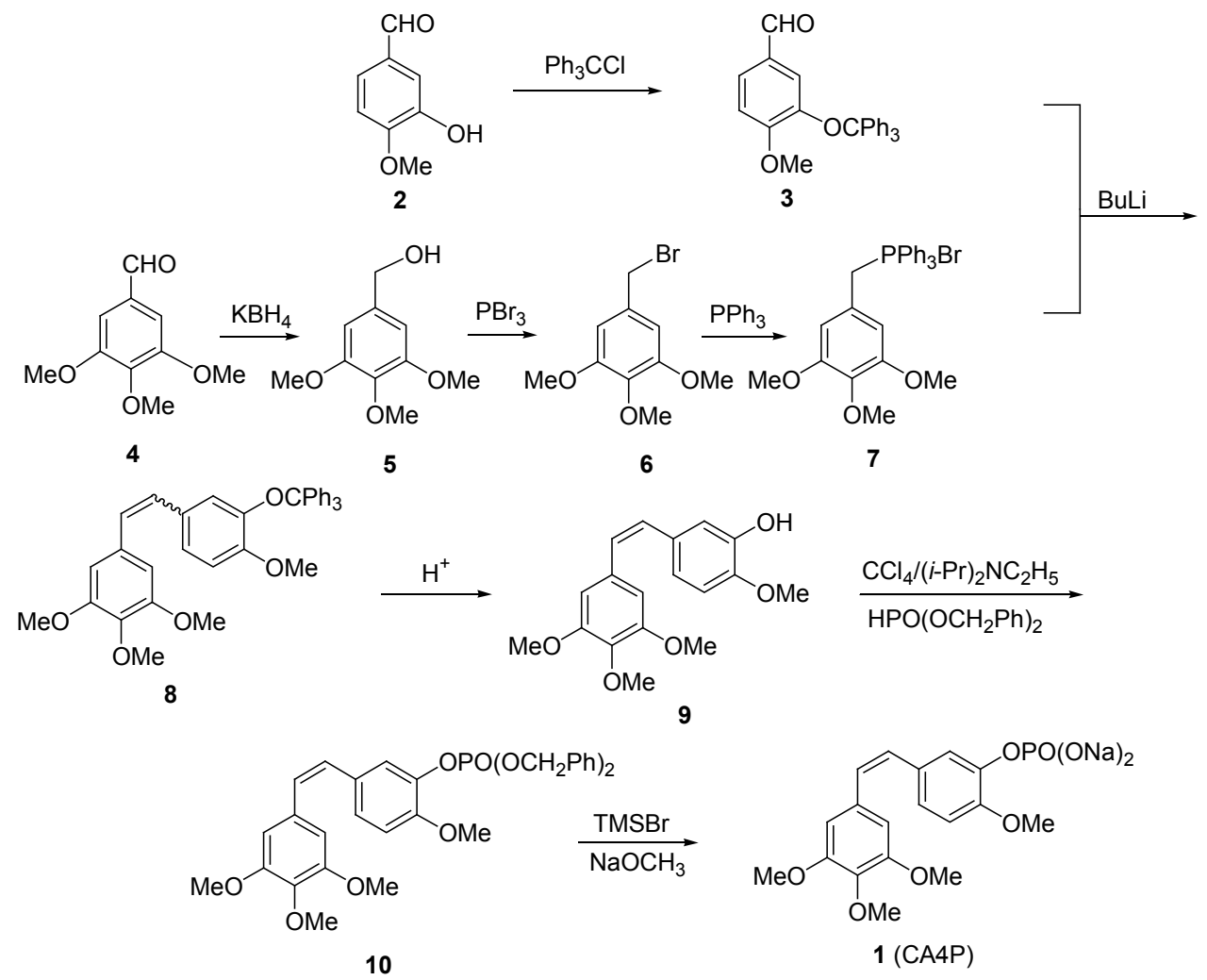

Scheme 1 
左右的反式异构体, 一般需要过柱的方法 ${ }^{[11]}$ 将其去除. 本文采用了乙醇-石油醚混合溶剂重结晶的方法, 非常 方便地将化合物 9 的纯度提高到 $98 \%$ 以上.

最后一步脱茮基成钠盐反应中, 反应温度对反应的 收率和产品的质量有非常大的影响. 反应温度过高, 反 式异构体的含量明显增加. 反应温度过低, 不利于工业 化生产. 为此确定反应的最佳温度为 $-5 \sim 0{ }^{\circ} \mathrm{C}$.

\section{2 结论}

以 3,4,5-三甲氧基苯甲醛为原料, 经还原、溴代、 成鏻盐、Wittig 反应、脱三苯甲基保护基、与亚磷酸二 芐酯成酯、脱芐基成钠盐等步骤, 合成制得康布瑞汀磷 酸酯二钠盐. 所得康布瑞汀磷酸酯二钠盐(1), HPLC 纯 度达 $99.5 \%$. 总收率为 $43.0 \%$. 经优化后的康布瑞汀磷 酸酯二钠盐的合成工艺, 原料价廉易得、工艺操作简便, 原料成本大幅度下降. 该工艺已实现了中试规模的工业 化生产.

\section{3 实验部分}

\section{1 仪器与试剂}

Brucker AM500 (500 MHz)核磁共振仪, TMS 为内 标; GC 测定含量, 仪器为岛津 SH IMADZU. HPLC 测定 含量, 仪器为岛津 SH IMADZU(进样洜: LC - 10AT, 检 测器: SPD210A).

异香草醛、三苯甲基氯、3,4,5-三甲氧基苯甲醛、 三溴化磷、三氯化磷、嗍氢化钾、三苯基膦、正丁基锂、 二异丙基乙基胺、三甲基溴硅烷、4-二甲基氨基吡啶、 甲醇钠溶液, 以上原料均为国产市售工业品. 芐醇、二 甲苯胺、三乙胺、乙酸乙酯、正庚烷、石油醚、二氯甲 烷、无水乙醇、四氢呋喃、乙腈、浓盐酸、氢氧化钠、 碳酸氢钠、无水硫酸镁、硅胶、磷酸二氢钾、四氯化碳、 甲醇、丙酮, 以上试剂均为国产分析纯或化学纯. 反应 所用的溶剂均经过无水处理.

\section{2 实验方法}

\subsection{1 亚磷酸二華酯(DBP)的合成}

在 $5000 \mathrm{~mL}$ 的三口瓶中, 加入干燥过的甲苯 2500 $\mathrm{mL}$ 、三氯化磷 $(343.4 \mathrm{~g}, 2.5 \mathrm{~mol})$, 氮气保护下搅拌降温 到 $0{ }^{\circ} \mathrm{C}$. 滴加芐醇 $(541 \mathrm{~g}, 5.0 \mathrm{~mol})$ 、二甲苯胺 $(655 \mathrm{~g}, 5.4$ $\mathrm{mol}$ )和甲苯 $600 \mathrm{~mL}$ 的混合液, 滴完继续反应半小时. 继 续滴加芐醇 $(270 \mathrm{~g}, 2.5 \mathrm{~mol})$, 滴加完搅拌过夜. 加入 $1000 \mathrm{~mL}$ 水, 分液, 甲苯层依次用水 $1000 \mathrm{~mL}, 11 \%$ 氨水 $1000 \mathrm{~mL}$, 水 $1000 \mathrm{~mL}$ 洗涤. 有机相经干燥、过滤和浓缩 得到油状液体粗品 $700 \mathrm{~g}$ (文献值 ${ }^{[15]} 640 \mathrm{~g}$ ). 粗品用硅胶 柱快速过柱, 得到 DBP 产品 $500 \mathrm{~g}, \mathrm{GC}$ 含量 $85 \%$. 折纯
收率 $65 \%$.

\subsubsection{3-三苯甲氧基-4-甲氧基苯甲醛(3)的合成}

在 $500 \mathrm{~mL}$ 四口瓶中加入异香草醛 $(50 \mathrm{~g}, 0.329$ $\mathrm{mol}) 、 三$ 苯甲基氯(103.7 g, $0.372 \mathrm{~mol})$ 和干燥四氢呋喃 $(180 \mathrm{~mL})$. 氩气保护, 在 $40{ }^{\circ} \mathrm{C}$ 下搅拌 $30 \mathrm{~min}$. 向反应体 系中滴加三乙胺 $(64.8 \mathrm{~mL}, 0.468 \mathrm{~mol}), \mathrm{TLC}$ 跟踪反应结 束后，向反应体系中加入水 $(200 \mathrm{~mL})$, 降温到 $20 \sim 25$ ${ }^{\circ} \mathrm{C}$. 加入乙酸乙酯 $(54 \mathrm{~mL})$ 、正庚烷 $(108 \mathrm{~mL})$, 生成有颗 粒状浅黄色固体，抽滤，所得固体依次用水 $(90 \mathrm{~mL} \times 2)$ 、 乙酸乙酯-正庚烷 (体积比 $=1: 2,54 \mathrm{~mL} \times 2$ )、石油醚 $(55$ $\mathrm{mL} \times 2$ ) 洗涤, 真空干燥, 得到淡黄色固体 $101.6 \mathrm{~g}$, HPLC 含量 98.1\%. 收率 78.3\% (文献值 ${ }^{[13]} 65 \%$ 75\%). ${ }^{1} \mathrm{H}$ NMR $\left(\mathrm{CDCl}_{3}, 500 \mathrm{MHz}\right) \delta: 3.62(\mathrm{~s}, 3 \mathrm{H}), 6.68 \sim 7.48$ (m, 18H), $9.54(\mathrm{~s}, 1 \mathrm{H})$.

\subsubsection{3,4,5-三甲氧基芐溴三苯基鏻盐(7)的合成}

将 3,4,5-三甲氧基苯甲醛(86.9 g, $0.443 \mathrm{~mol}$ )溶于无 水乙醇 $(400 \mathrm{~mL})$ 中, $25{ }^{\circ} \mathrm{C}$ 下分批加入硼氢化钾 $(7.2 \mathrm{~g}$, $0.134 \mathrm{~mol}), \mathrm{TLC}$ 跟踪反应结束后, 加入水 $(12.5 \mathrm{~mL})$. 浓 缩得到淡黄色油状物, 加入水 $(75 \mathrm{~mL})$ 和二氯甲烷 $(100$ $\mathrm{mL})$, 分层, 水层用二氯甲烷 $(25 \mathrm{~mL} \times 2)$ 萃取. 合并有机 层, 饱和食盐水 $50 \mathrm{~mL} \times 2$ 洗至近中性, 干燥、过滤、浓 缩得到淡黄色油状液体(化合物 5) $89 \mathrm{~g}$, 含量 $98 \%$ 以上, 收率 100\%.

在 $500 \mathrm{~mL}$ 的反应瓶中, 将油状化合物 $5(45 \mathrm{~g}$, $0.227 \mathrm{~mol}$ )溶于 $230 \mathrm{~mL}$ 甲苯, 搅拌, 降温至 $-5 \sim 0{ }^{\circ} \mathrm{C}$, 滴加三溴化磷 $(11 \mathrm{~mL}, 30.8 \mathrm{~g}, 0.323 \mathrm{~mol}), 0{ }^{\circ} \mathrm{C}$ 继续搅拌 至反应完全. 加水 $(15 \mathrm{~mL})$ 分层, 有机层用饱和碳酸氢钠 $(25 \mathrm{~mL} \times 3)$ 洗涤至 $\mathrm{pH}=7.5 \sim 8$, 干燥、过滤, 滤液中加 入三苯基膦 $(61 \mathrm{~g}, 0.233 \mathrm{~mol}), 25{ }^{\circ} \mathrm{C}$ 搅拌 $48 \mathrm{~h}$. 过滤、真 空干燥, 得到的粗品用无水乙醇重结晶, 得到白色结晶 固体 $85 \mathrm{~g}, \mathrm{HPLC}$ 含量 $98.4 \%$. 收率 $80.0 \%$ (文献值 ${ }^{[14]}$ 69.3\%). ${ }^{1} \mathrm{H}$ NMR $\left(\mathrm{CDCl}_{3}, 500 \mathrm{MHz}\right) \delta: 3.52(\mathrm{~s}, 6 \mathrm{H}), 3.77$ (s, 3H), 5.39 (s, 2H), 6.47 (s, 2H), 6.68 7.71 (m, 15H).

3.3.4 3,4,5, 4'-四甲氧基-3'-三苯甲氧基-二苯乙烯 $(8)$ 的合成

在 $500 \mathrm{~mL}$ 四口瓶中, 在氩气保护下, 加入 3,4,5-三 甲氧基芐溴三苯基鏻盐 $7(65 \mathrm{~g}, 0.122 \mathrm{~mol})$ 、无水四氢呋 喃 $(112 \mathrm{~mL})$. 在 $-20 \sim-15{ }^{\circ} \mathrm{C}$, 滴加正丁基锂的正己烷 溶液 $\left(2.4 \mathrm{~mol} \cdot \mathrm{L}^{-1}, 61 \mathrm{~mL}, 0.146 \mathrm{~mol}\right)$. 滴完后搅拌 60 $\min$, 滴加化合物 $3(48.8 \mathrm{~g}, 0.122 \mathrm{~mol})$ 的四氢呋喃(220 $\mathrm{mL}$ )溶液, 滴完后自然升温至 $25{ }^{\circ} \mathrm{C}$, 搅拌过夜. 次日反 应完毕后, 将温度降至 $-5{ }^{\circ} \mathrm{C}$, 加入饱和食盐水 280 $\mathrm{mL}$. 分液, 有机层用饱和食盐水 $(100 \mathrm{~mL} \times 2)$ 洗涤, 干 燥、浓缩, 残液加入 3 倍体积的无水乙醇摚拌, 抽滤、 真空干燥, 得白色固体 $56 \mathrm{~g}$, HPLC 含量 99\%(顺反比例 
为 $=87: 12$ ), 收率 $83 \%$ (文献值 ${ }^{[13]} 71 \% \sim 92 \%$, 顺反比 例为 $60: 40 \sim 75: 25)$.

\subsubsection{3,4,5,4'-四甲氧基-3'-羟基一顺式-二苯乙烯(9) 的合成}

在 $200 \mathrm{~mL}$ 的三口瓶中, 加入化合物 $8(55 \mathrm{~g}, 0.099$ $\mathrm{mol})$ 、乙醇 $60 \mathrm{~mL}$. 在 $25{ }^{\circ} \mathrm{C}$ 水浴下滴加 $37 \%$ 浓盐酸 $(90$ $\mathrm{mL}), 1 \mathrm{~h}$ 滴完后. 继续反应 $3 \mathrm{~h}$. 加水 $(55 \mathrm{~mL})$ 中止反应. 有固体析出, 过滤、真空干燥, 得产物 $35.0 \mathrm{~g}$, HPLC 含 量 $82 \%$. 折纯收率 $92 \%$. (文献值 ${ }^{[13]} 45 \% \sim 65 \%$, 无纯度 数据.) 不需提纯可直接用于下一步反应.

将上述粗品用乙醇一石油醚 $(V: V=1: 2)$ 混合溶剂 重结晶, 得到化合物 9 纯品, HPLC 纯度 99.8\%. ${ }^{1} \mathrm{H}$ NMR $\left(\mathrm{CDCl}_{3}, 500 \mathrm{MHz}\right) \delta: 3.69(\mathrm{~s}, 6 \mathrm{H}), 3.84(\mathrm{~s}, 3 \mathrm{H}), 3.85(\mathrm{~s}$, $3 \mathrm{H}), 5.58(\mathrm{~s}, 1 \mathrm{H}), 6.41(\mathrm{~d}, J=12.0 \mathrm{~Hz}, 1 \mathrm{H}), 6.46(\mathrm{~d}, J=$ $12.0 \mathrm{~Hz}, 1 \mathrm{H}), 6.53$ (s, 2H), $6.73(\mathrm{~d}, J=8.5 \mathrm{~Hz}, 1 \mathrm{H}), 6.79$ $(\mathrm{dd}, J=8.5,2.0 \mathrm{~Hz}, 1 \mathrm{H}), 6.92(\mathrm{~d}, J=2.0 \mathrm{~Hz}, 1 \mathrm{H})$.

3.3.6 3,4,5,4'-四甲氧基一顺式-二苯乙烯-3'- $O$-磷酸二 苯酯 $(\mathbf{1 0})$ 的合成

在 $500 \mathrm{~mL}$ 四口烧瓶中, 通入氩气, 加入 9 (折纯后 $20 \mathrm{~g}, 0.0632 \mathrm{~mol}$ )、干燥乙腈 $150 \mathrm{~mL}$ 搅拌溶解, 冷却到 $-25{ }^{\circ} \mathrm{C}$, 依次加入干燥四氯化碳(12.7 g)、二异丙基乙 基胺(12.3 g)和 4-二甲基氨基吡啶 $(0.8 \mathrm{~g})$, 缓慢滴加亚磷 酸二芐酯 $(18.2 \mathrm{~g}, 0.0695 \mathrm{~mol})$ 与干燥乙腈 $(50 \mathrm{~mL})$ 混合溶 液, 控制温度在 $-10{ }^{\circ} \mathrm{C}$ 以下, $2 \mathrm{~h}$ 内滴加完毕. TLC 跟踪 显示原料点消失后, 继续反应 $3.5 \mathrm{~h}$. 自然升温到 $20 \sim 25$ ${ }^{\circ} \mathrm{C}$, 加入 $0.5 \mathrm{~mol} \cdot \mathrm{L}^{-1}$ 磷酸二氢钾 $(52.5 \mathrm{~mL})$ 中止反应. 水 层用乙酸乙酯 $(300 \mathrm{~mL} \times 2)$ 萃取, 合并有机层, 依次用蒸 馏水 $(250 \mathrm{~mL} \times 3)$ 、食盐水 $(250 \mathrm{~mL} \times 3)$ 洗涤至中性, 干 燥、浓缩得到油状物粗品 $50 \mathrm{~g}$.

将上述油状物粗品 $50 \mathrm{~g}$ 用无水乙醇和石油醚 $(V$ : $V=1: 1,150 \mathrm{~mL}$ )重结晶, 得到白色固体 $32 \mathrm{~g}$, 收率 $88 \%$. HPLC 含量 $98 \%$. ${ }^{1} \mathrm{H}$ NMR $\left(\mathrm{CDCl}_{3}, 500 \mathrm{MHz}\right) \delta$ : $3.68(\mathrm{~s}, 6 \mathrm{H}), 3.80(\mathrm{~s}, 3 \mathrm{H}), 3.77(\mathrm{~s}, 3 \mathrm{H}), 5.12 \sim 5.14(\mathrm{~m}$, $4 \mathrm{H}), 6.42 \sim 6.49(\mathrm{~m}, 4 \mathrm{H}), 6.79(\mathrm{~d}, J=8.5 \mathrm{~Hz}, 1 \mathrm{H}), 7.07(\mathrm{~s}$, 1H), 7.15 (s, 1H), 7.32 (s, 10H); ${ }^{31} \mathrm{P}$ NMR $\left(\mathrm{CDCl}_{3}, 202.5\right.$ MHz) $\delta:-2.83(\mathrm{~s})$.

3.3.7 3,4,5,4'-四甲氧基-顺式-二苯乙烯-3'- $O$-磷酸二 钠盐(1)的合成

在 $1000 \mathrm{~mL}$ 反应瓶中, 加入化合物 $\mathbf{1 0}(90 \mathrm{~g}, 0.16$ $\mathrm{mol})$ 、无水乙腈 $450 \mathrm{~mL}$, 氩气保护下, 冷却到 $-5^{\circ} \mathrm{C}$ 摚 拌, 快速滴加新蒸三甲基溴硅烷 $(59.4 \mathrm{~mL}, 0.45 \mathrm{~mol})(10$ $\min$ 左右滴完), 滴加完毕后搅拌 $30 \mathrm{~min} .-5{ }^{\circ} \mathrm{C}$ 下滴加
甲醇/甲醇钠溶液(浓度 $29.8 \%, 163 \mathrm{~g}, 0.90 \mathrm{~mol}$ ). 继续摚 拌 $30 \mathrm{~min}$, 加入丙酮-甲醇溶液 $(80 \mathrm{~mL}$, 体积比 $=1: 1)$, 常温摚拌过夜. 过滤、真空干燥, 得到磷酸酯二钠盐粗 品 $96 \mathrm{~g}$. 粗品经丙酮-水 $(V: V=5: 1)$ 混合溶剂重结晶 2 次, 真空干燥得到白色粉末固体 $58.8 \mathrm{~g}$, 收率 $80 \%$ (以化 合物 9 计算, 最后两步的收率为 $70.4 \%$, 文献[13]最后两 步的收率 $40 \% \sim 55 \%$ ), HPLC 含量 $99.5 \%$, 其它指标符合 质量要求. ${ }^{1} \mathrm{H}$ NMR $\left(\mathrm{D}_{2} \mathrm{O}, 500 \mathrm{MHz}\right) \delta: 3.64(\mathrm{~s}, 6 \mathrm{H}), 3.70$ (s, 3H), $3.81(\mathrm{~s}, 3 \mathrm{H}), 6.41(\mathrm{~d}, J=12.0 \mathrm{~Hz}, 1 \mathrm{H}), 6.56$ (s, 2H), $6.62(\mathrm{~d}, J=12.0 \mathrm{~Hz}, 1 \mathrm{H}), 6.73(\mathrm{~d}, J=8.5 \mathrm{~Hz}, 1 \mathrm{H})$, $6.80(\mathrm{~d}, J=8.5 \mathrm{~Hz}, 1 \mathrm{H}), 7.39(\mathrm{~s}, 1 \mathrm{H}) ;{ }^{13} \mathrm{C}$ NMR $\left(\mathrm{D}_{2} \mathrm{O}\right.$, $125.8 \mathrm{MHz}) \delta$ : $58.67,58.74,63.67,109.29,115.25$, $124.10,125.27,131.37,132.89,133.08,136.59,138.52$, 145.90, 151.90, 154.87; ${ }^{31} \mathrm{P}$ NMR $\left(\mathrm{D}_{2} \mathrm{O}, 202.5 \mathrm{MHz}\right) \delta$ : 3.25 (s) (与文献[13]一致).

\section{References}

[1] Zhang, X ; Zhang, J.-C. China Pharmacy 2007, 18(10), 779 (in Chinese).

(张璇, 张建存, 中国药房, 2007, 18(10), 779.)

[2] Hua, W.-T.; Du, D.-M.; Zhang, H.; Wu, Q.-H. CN 1465580, 2004 [Chem. Abstr. 2004, 142, 316955].

[3] He, Y.-J.; Zhang, J.; Liu, J.; He, W.-W.; Zhou, S.; Tian, X.; Xu, X.-P. West China J. Pharm. Sci. 2008, 23(6), 663 (in Chinese). (何应金, 张洁, 刘静, 何雯雯, 周莎, 田信, 徐小平, 华西药学 杂志, 2008, 23(6), 663.)

[4] Lippert, J. W. Bioorg. Med. Chem. 2007, 15, 605.

[5] Gill, S. G.; Grobelny, D.; Flynn, B. Org. Prep. Proc. Int. 2006, 38(6), 604.

[6] Yong, Z.-Q.; Xu, X.-P.; Chen, Y.-C.; Bao, X.; Weng, L.-L.; Zheng, H. Chin. Chem. Lett. 2006, 17(1), 23.

[7] Zou, Y.; Zhong, R.-Q.; Zhang, X.-J.; Zhou, Y.; Wang, Z.-X.; Lin, H.-Z., CN 1660745, 2005 [Chem. Abstr. 2005, 145, 83106].

[8] Yang, Z.-Q.; Qiu, G.-F.; Liang, S.-C.; Hu, X.-M. Chin. J. Mod. Appl. Pharm. 2005, 22(3), 210 (in Chinese).

(杨兆琪, 邱国福, 粱淑彩, 胡先明, 中国现代应用药学, 2005, 22(3), 210.)

[9] Li, P.; Zeng, Y.-J.; Deng, J.-B.; Li, H.-Y.; Zhou, W.; Chen, C.-H. CN 101220054A, 2008 [Chem. Abstr. 2008, 149, 246648].

[10] Ma, S.-N.; He, Q.-K.; Cai, Z.-W.; Zuo, X.-Y. CN 1737004, 2006 [Chem. Abstr. 2006, 146, 145878].

[11] Peng, H.-W.; Yang, W.; Zhao, B.; Dong, Z.-Y.; Deng, J.-B.; Zhou, W. CN 101323626A, 2008.

[12] Zou, Y.; Huang, W.-M. CN 101186620, 2008 [Chem. Abstr. 2008, 149, 68779].

[13] Faye, S.; Jonathan, G.; Reem, H.; John, H. WO 2002006279, 2002 [Chem. Abstr. 2002, 136, 134622].

[14] Liu, S.-X.; Shen, P.-Q. Qilu Pharm. Affairs 2007, 26(2), 107 (in Chinese). (刘双喜, 沈配倩, 齐鲁药事, 2007, 26(2), 107.)

[15] Schlitzer, M. Synthesis 2007, 3553. 